\title{
EW and QCD One-Loop Amplitudes with RECOLA
}

\author{
S. Actis \\ Paul Scherrer Institut ${ }^{*}$
}

\section{A. Denner}

Universität Würzburg

E-mail: denner@physik.uni-wuerzburg.de

\section{Hofer ${ }^{\dagger}$}

Universität Würzburg

E-mail: Ihofer@ifae.es

\section{A. Scharf}

Universität Würzburg

E-mail: ascharf@physik.uni-wuerzburg.de

\section{S. Uccirati}

Torino University and INFN

E-mail: ucciratieto.infn.it

We present the computer code RECOLA for the computation of EW and QCD amplitudes in the Standard Model at next-to-leading order. One-loop amplitudes are represented as linear combinations of tensor integrals whose coefficients are calculated by means of recursive relations similar to Dyson-Schwinger equations. A novel treatment of colour enables us to recursively construct the colour structure of the amplitude efficiently. RECOLA is linked with the library COLLIER for the computation of the tensor integrals.

11th International Symposium on Radiative Corrections (Applications of Quantum Field Theory to Phenomenology) (RADCOR 2013),

22-27 September 2013

Lumley Castle Hotel, Durham, UK

\footnotetext{
${ }^{*}$ Former affiliation

${ }^{\dagger}$ Present address: Universitat Autònoma de Barcelona

† Speaker.
} 


\section{Introduction}

All the particles predicted by the Standard Model have been confirmed by experiments, yet many sectors of this successful theory have to be precisely investigated. The description of high energy processes is based on pertubation theory, and accurate theoretical predictions require a detailed understanding beyond Born approximation. At a hadron collider as the LHC, QCD corrections are known to be large, but also electroweak (EW) corrections can have an important impact. Indeed the high energies attained by the LHC allow to collect data in phase-space regions where the effects of logarithms of EW origin become sizable. In particular cases like e.g. Higgs production in vector-boson fusion the EW corrections can be of the same order of magnitude as QCD corrections [1]. Finally, photon emission creates mass-singular logarithms which again lead to large contributions. Therefore a proper theoretical description of LHC physics requires next-to-leadingorder (NLO) computations of multiparticle processes (with five, six, or more external legs) in the full SM (including EW corrections).

While QCD corrections have been calculated in the last years for many processes, in the EW sector the situation is less satisfactory. Usually EW one-loop computations are more involved than QCD ones and often rely on codes created "ad hoc" to compute specific processes, whose generalization to other processes is tedious. Moreover the computation of NLO corrections for the elementary scattering is not the end of the story and many other issues have to be considered in hadronic processes (such as multi-channel Monte Carlos, a proper treatment of real emission, convolution with the parton distribution functions, parton shower, etc.). For this reason the creation of general and automatized codes for the computation of NLO partonic matrix elements is highly desirable. In the past years many groups have concentrated their efforts to make such calculations feasible, and a lot of codes have appeared $[2,3,4,5,6,7,8]$ with a high level of automatization and impressive performances, however restricting their range of applicability mostly to the QCD sector of the SM.

For this reason we have developed RECOLA, a generator of tree- and one-loop amplitudes in the full SM [9]. The code overcomes the difficulties in automatizing efficiently computations based on Feynman diagrams, choosing an alternative approach which extends to one-loop amplitudes the known recursion relations for tree-level amplitudes. The idea, originally proposed by Andreas van Hameren in Ref. [10] for gluonic amplitudes, is to write one-loop amplitudes as linear combinations of tensor integrals and compute recursively the coefficients of such a decomposition. The tensor integrals themselves are then computed by linking the code to external libraries.

\section{Tree-level recursion relations}

The tree-level recursive algorithm is inspired by the Dyson-Schwinger equations [11], using off-shell currents as basic building blocks. Let us consider a process with $L$ external legs, written in the form $P_{1}+\cdots+P_{L-1}+P_{L} \rightarrow 0$, where we select one particle to be the last $\left(P_{L}\right)$ while the others are called primary. The off-shell current

$$
w\left(P, \mathscr{C}, B_{\left\{l_{1}, \ldots, l_{n}\right\}}\right)={ }^{P} \cdot
$$

is defined as the amplitude made of $n$ of the $L-1$ primary on-shell particles with labels $\left\{l_{1}, \ldots, l_{n}\right\}$ and the off-shell particle $P$ (carrying colour labeled by $\mathscr{C}$ ). The off-shellness of the particle $P$ 
(depicted by a dot) implies that its wave function is not included and, for $n>1$, replaced by its propagator. The off-shell current $w$ has a Lorentz structure of a scalar, spinor or vector according to the type of particle $P$. For $n=1$ the off-shell currents of the external legs coincide with their wave functions. The labels $\left\{l_{1}, \ldots, l_{n}\right\}$ are expressed through one tag number $B_{\left\{l_{1}, \ldots, l_{n}\right\}}$, obtained using the binary notation of Ref. [12]: each external particle $P_{i}$ is labeled by the tag number $B_{\{i\}}=2^{i-1}$ (i.e. $B_{\{1\}}=1, B_{\{2\}}=2, B_{\{3\}}=4, B_{\{4\}}=8, \ldots$ ), while the tag number of the internal currents is simply obtained by summing the tag numbers of the external currents contributing to it (for example $B_{\{1,2,4\}}=B_{\{1\}}+B_{\{2\}}+B_{\{4\}}=1+2+8=11$ ).

The practical advantage of using off-shell currents is that they can be computed recursively using the Dyson-Schwinger equation, which for the SM reads:

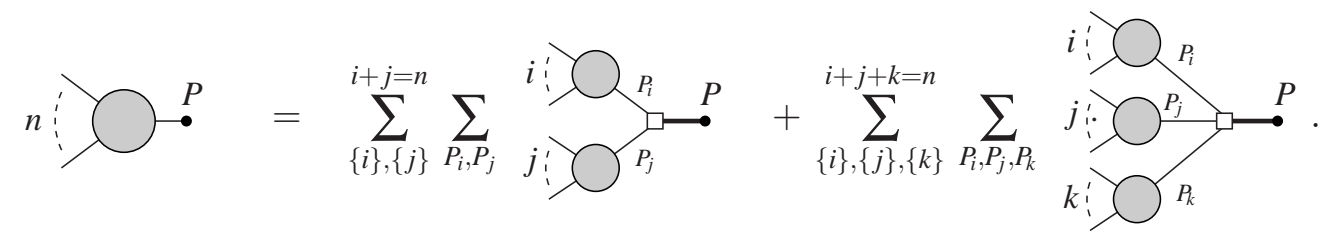

Each term of the sums represents a "branch", which is obtained by multiplying the generating currents with the interaction vertex, marked by the small box, and the propagator of $P$, marked by the thick line. The recursion procedure consists in using the Dyson-Schwinger equation starting from $n=2$, for whatever allowed particle $P$, obtaining the 2-leg currents:

$$
\bigcirc \cdot=\gamma
$$

This result is then used to compute the 3-leg currents, again for all possible $P$ :

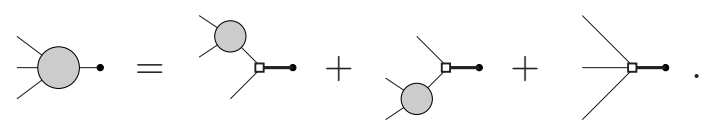

Proceeding in this way, one can compute recursively all currents for $n \leq L-1$. For the last step, i.e. for $n=L-1$, not all currents are needed, but just the one with the particle $P$ coinciding with the antiparticle $\bar{P}_{L}$ of the last particle. The amplitude $\mathscr{A}$ is then obtained by multiplying this unique last current with the inverse of the propagator of $\bar{P}_{L}$ and with its wave function:

$$
\mathscr{A}=L-1 \bigcirc \stackrel{P}{L}^{\bar{P}_{2}} \times\left(\text { propagator of } \bar{P}_{L}\right)^{-1} \times \bullet \bar{P}_{L}
$$

The advantage of the recursion procedure with respect to the usual approach based on Feynman diagrams is the possibility to avoid recomputing identical sub-graphs contributing to different diagrams; furthermore, each current being the sum of many sub-graphs, the number of generated objects which are passed to the next step of the recursion is reduced with respect to a "diagram by diagram" procedure.

\section{One-loop recursion relations}

Every one-loop amplitude can be written as a linear combination of tensor integrals (TIs):

$$
\mathscr{A}=\sum_{t} c_{\mu_{1} \cdots \mu_{r_{t}}}^{(t)} T_{(t)}^{\mu_{1} \cdots \mu_{r_{t}}}
$$

The tensor coefficients (TCs) $c_{\mu_{1} \cdots \mu_{r_{t}}}^{(t)}$ do not depend on the loop momentum $q$, which is present only in the TIs: 


$$
T_{(t)}^{\mu_{1} \cdots \mu_{r_{t}}}=\frac{(2 \pi \mu)^{4-D}}{\mathrm{i} \pi^{2}} \int \mathrm{d}^{D} q \frac{q^{\mu_{1}} \cdots q^{\mu_{r_{t}}}}{D_{0}^{(t)} \cdots D_{k_{t}}^{(t)}}, \quad D_{k_{t}}^{(t)}=\left(q+p_{k_{t}}^{(t)}\right)^{2}-\left(m_{k_{t}}^{(t)}\right)^{2} .
$$

The key of an automatized computation of one-loop amplitudes for complicated processes is to find an efficient procedure to compute the TCs $c_{\mu_{1} \cdots \mu_{r_{t}}}^{(t)}$. Our approach to solve this problem starts from the work of van Hameren [10], which proposed a way to generalize the Dyson-Schwinger recursion relation to one-loop gluon amplitudes in QCD. We have further developed this approach to deal with the full SM. Thanks to the correspondence between a one-loop diagram with $L$ external legs and a tree diagram with $L+2$ external legs (obtained by cutting one of the loop lines), one can associate with the loop process $P_{1}+\cdots+P_{L} \rightarrow 0$ a family of tree processes with two more legs $P_{1}+\cdots+P_{L}+P_{0}+\bar{P}_{0} \rightarrow 0$, for any particle $P_{0}$ of the SM. This correspondence is however not unique and selection rules have to be applied to identify the tree diagram associated with a given one-loop diagram. The problem basically arises from the fact that the loop diagram can be cut at any of its loop lines and that we can run along the loop clockwise or counterclockwise. As a simple example we consider a three-point function at one loop and find six corresponding tree level diagrams:

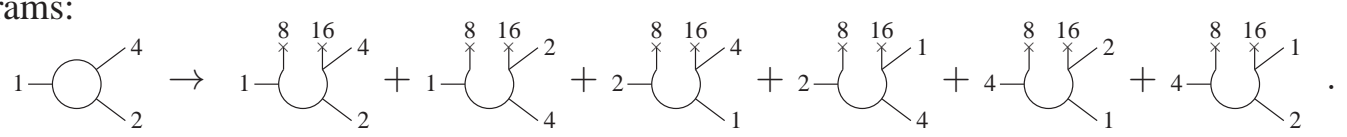

The tree diagrams have been drawn in such a way, that one can easily identify the original sequence of the loop lines (called "loop flow"), starting from the external loop leg with tag number $2^{L}$ and ending with the external loop leg with tag number $2^{L+1}$. The selection rules must discard the redundant contributions and be simple enough to be translated from the diagrammatic representation into the language of off-shell currents. Two rules accomplishing this are:

1) The current containing the first external line enters the loop flow first.

2) The 3 currents containing the external legs with the 3 smallest binary numbers enter the loop flow in fixed order (for example given by the ascending order of the 3 binaries).

Applying the first rule to our example, we discard 4 redundant diagrams:

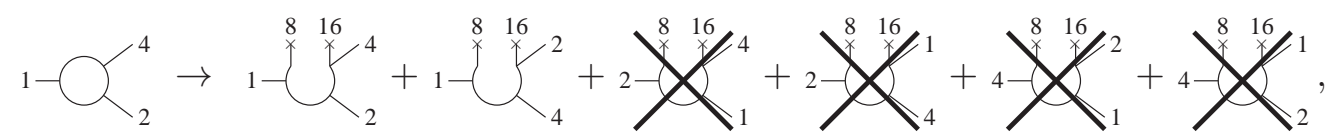

while the second rule excludes the last unwanted contribution

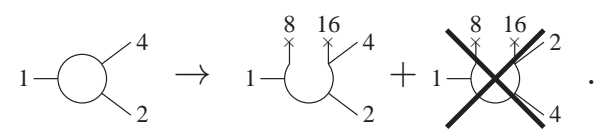

Having reduced the formal generation of the one-loop amplitude to the generation of a set of tree-level processes, we can build the "loop off-shell currents" in a similar way as the tree-level currents, by means of a recursion relation:

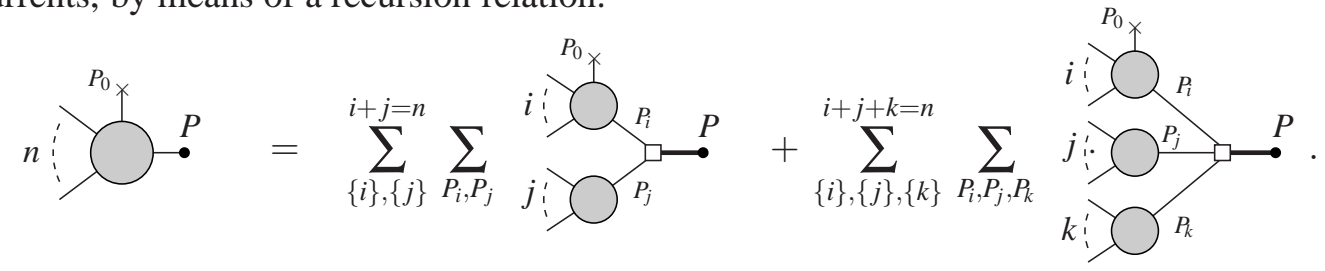

Formally the relation is the same as at tree level, with the additional leg $P_{0}$ (at loop level the particle $\bar{P}_{0}$ is chosen as last). The main difference comes actually form the fact that every loop current 
contains a dependence on the integration momentum $q$, generated by the Feynman rules for the vertex and the propagator. Working in the 't Hooft-Feynman gauge in the SM, the $q$-dependence of $($ vertex $) \times($ propagator $)$ takes the form

$$
(\text { vertex }) \times(\text { propagator })=\frac{a_{\mu} q^{\mu}+b}{(q+p)^{2}-m^{2}} .
$$

This $q$-dependence determines through repeated application of the recursion relation the structure of the loop currents, which after $k$ steps gets a $q$-dependent structure of the type:

$$
\text { loop current }(q)=\sum_{r=0}^{k} a_{\mu_{1} \cdots \mu_{r}}^{(k, r)} \frac{q^{\mu_{1}} \cdots q^{\mu_{r}}}{\prod_{h=1}^{k}\left[\left(q+p_{h}\right)^{2}-m_{h}^{2}\right]},
$$

where $k$ is the number of $q$-dependent propagators and $r$ is the rank of each term of the linear combination. The $q$-dependence of the loop currents in Eq. (3.5) has the same structure as the one-loop amplitude of Eq. (3.1). In particular the coefficients $a_{\mu_{1} \cdots \mu_{r}}^{(k, r)}$ that we get at the last step of the procedure coincide with the TCs $c_{\mu_{1} \cdots \mu_{r_{t}}}^{(t)}$. Therefore we use the recursive relation in Eq. (3.3) to compute at each step the coefficients $a_{\mu_{1} \cdots \mu_{r}}^{(k, r)}$ of the loop current, keeping track of the corresponding loop propagators. This is done by defining the $q$-independent loop off-shell current

$$
w_{i_{k}}\left(P, \mathscr{C}, B,\left\{p_{1}, \ldots, p_{k}\right\},\left\{m_{1}, \ldots, m_{k}\right\}\right)=
$$

where the sequences of off-set momenta $\left\{p_{1}, \ldots, p_{k}\right\}$ and of masses $\left\{m_{1}, \ldots, m_{k}\right\}$ of the propagators link each $w_{i_{k}}$ with its $q$ structure and at the end of the procedure allow to relate each TC to its TI. The tensorial index $i_{k}$ of the loop current $w_{i_{k}}$ is a short-hand notation to express the coefficients $a_{\mu_{1} \cdots \mu_{r}}^{(k, r)}$ for all values of $r$ and $\mu_{1} \cdots \mu_{r}$ :

$$
i_{k}=0 \rightarrow w_{i_{k}}=a^{(k, 0)}, \quad i_{k}=1, \ldots, 4 \rightarrow w_{i_{k}}=a_{\mu_{1}}^{(k, 1)}, \quad i_{k}=5, \ldots, 14 \rightarrow w_{i_{k}}=a_{\left\{\mu_{1} \mu_{2}\right\}}^{(k, 2)},
$$

where we considered that only symmetric combinations of the indices $\mu_{1}, \ldots, \mu_{r}$ give non-vanishing contributions.

In order to apply the recursive relation in Eq. (3.3), it remains to understand which singleparticle currents have to be associated with the new particles $P_{0}$ and $\bar{P}_{0}$. This is done by introducing for fermions and vector bosons a suitable set of spinors $\psi_{i}$ and polarization vectors $\varepsilon_{i}^{\mu}$, in such a way that the contraction originally contained in the loop diagrams can be reproduced:

$$
\left(\psi_{i}\right)_{\alpha}=\left(\bar{\psi}_{i}\right)_{\alpha}=\delta_{i \alpha} \quad \text { with } \quad \sum_{i=1}^{4}\left(\bar{\psi}_{i}\right)_{\alpha}\left(\psi_{i}\right)_{\beta}=\delta_{\alpha \beta}, \quad \varepsilon_{i}^{\mu}=\delta_{i}^{\mu} \quad \text { with } \quad \sum_{i=1}^{4} \varepsilon_{i}^{\mu} \varepsilon_{i}^{v}=\delta^{\mu v}
$$

where $i$ denotes the "polarization", and $\alpha, \beta$ and $\mu, v$ are spinor and Lorentz indices, respectively.

\section{Treatment of colour}

We use the colour-flow representation introduced in Ref. [13], where the conventional 8 gluon fields $A_{\mu}^{a}$ are replaced by a $3 \times 3$ matrix $\left(\mathscr{A}_{\mu}\right)_{j}^{i}=\frac{1}{\sqrt{2}} A_{\mu}^{a}\left(\lambda^{a}\right)_{j}^{i}$ with the trace condition $\sum_{i}\left(\mathscr{A}_{\mu}\right)_{i}^{i}=0$. Quarks and antiquarks maintain the usual colour index $i=1,2,3$, while gluons get a pair of indices $i, j=1,2,3$. The Feynman rules in this representation are in practice obtained by multiplying all 
gluon lines by $\left(\lambda^{a}\right)_{j}^{i}$ in the standard Feynman rules. Using the algebra of the Gell-Mann matrices, the colour part of the new Feynman rules consists simply in combinations of Kronecker $\delta$ s. For example one gets:

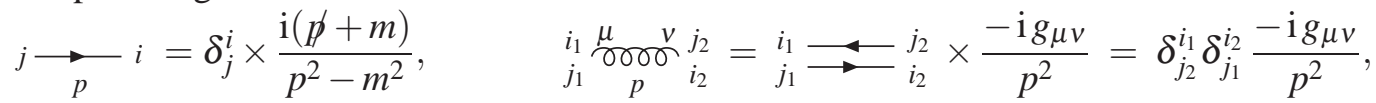

$$
\begin{aligned}
& \varlimsup_{j_{2}}^{i_{1}} \nearrow_{i_{i_{3}}}^{j_{j_{3}}}=\left({ }_{j_{2}}^{i_{1}} \backslash{ }_{i_{3}}^{j_{3}}-\frac{1}{N_{\mathrm{c}}}{ }_{j_{2}}^{i_{1}} \backslash-i_{i_{3}}^{j_{3}}\right) \times \frac{\mathrm{i} g_{\mathrm{s}}}{\sqrt{2}} \gamma^{\mu}=\left(\delta_{j_{3}}^{i_{1}} \delta_{j_{2}}^{i_{3}}-\frac{1}{N_{\mathrm{c}}} \delta_{j_{2}}^{i_{1}} \delta_{j_{3}}^{i_{3}}\right) \frac{\mathrm{i} g_{\mathrm{s}}}{\sqrt{2}} \gamma^{\mu}
\end{aligned}
$$

As a consequence, in the colour-flow representation the colour structure of the amplitude for a process with $k$ external gluons and $m$ external quark-antiquark pairs can be written as a linear combination of all possible products of Kronecker $\delta$ s:

$$
\mathscr{A}_{\beta_{1}, \ldots, \beta_{n}}^{\alpha_{1}, \ldots, \alpha_{n}}=\sum_{P\left(\beta_{1}, \ldots, \beta_{n}\right)} \delta_{\beta_{1}}^{\alpha_{1}} \cdots \delta_{\beta_{n}}^{\alpha_{n}} \mathscr{A}_{P}, \quad n=k+m,
$$

where we do not pose any restriction on the permutations $P\left(\beta_{1}, \ldots, \beta_{n}\right)$ to be considered. At this point basically two different strategies are possible for the computation of the sum over colours of the squared amplitude. In a framework based on colour-dressed amplitudes, all $N_{c}^{2 n}$ amplitudes $\mathscr{A}_{\beta_{1}, \ldots, \beta_{n}}^{\alpha_{1}, \ldots, \alpha_{n}}$ are computed (i.e. for all possible colour indices $\alpha_{1}, \ldots, \alpha_{n}, \beta_{1}, \ldots, \beta_{n}$ ) and the summed squared amplitude is given by

$$
\mathscr{A}^{2}=\sum_{\alpha_{1} \ldots \alpha_{n}, \beta_{1}, \ldots, \beta_{n}}\left(\mathscr{A}_{\beta_{1} \cdots \beta_{n}}^{\alpha_{1} \cdots \alpha_{n}}\right)^{*} \mathscr{A}_{\beta_{1} \cdots \beta_{n}}^{\alpha_{1} \cdots \alpha_{n}}
$$

Alternatively one can decide to use the decomposition of Eq. (4.2) and work directly with the $n$ ! "structure-dressed" amplitudes $\mathscr{A}_{P}$ of the colour structures $\delta_{\beta_{1}}^{\alpha_{1}} \cdots \delta_{\beta_{n}}^{\alpha_{n}}$. In this approach the summed squared amplitude is obtained by summing over the permutations according to

$$
\mathscr{A}^{2}=\sum_{P, P^{\prime}} \mathscr{A}_{P}^{*} C_{P P^{\prime}} \mathscr{A}_{P^{\prime}}
$$

where the coefficients $C_{P P^{\prime}}$ are trivially calculated (and simply given by positive powers of $N_{c}$ ). This second strategy reduces the number of amplitudes to compute and is essentially the starting point of the known approach based on colour-ordered amplitudes (which aims to reduce the number of amplitudes even more). Our implementation is slightly different. We assign a colour structure (labeled by the index $\mathscr{C}$ ) to each off-shell current and derive rules to build at each step of the recursion procedure the colour structure of the outgoing current from the colour structures of the incoming ones. In this way the colour structure of the amplitude is automatically generated, all structure-dressed amplitudes $\mathscr{A}_{P}$ are computed simultaneously in one recursive procedure, and structure-dressed currents which contribute at intermediate steps to several $\mathscr{A}_{P}$ are computed just once. Moreover one can further optimize the computation of currents which only differ by the colour structure, by recomputing just their colour coefficient instead of the whole current.

\section{Features of RECOLA}

The code RECOLA is structured in two parts: the generation of the recursion procedure (to be run once) and the computation of the currents (to be run at each phase-space point). In the generation procedure, the skeleton of the recursion procedure is created, assigning an index to each 


\begin{tabular}{|c|c|c|c|c|}
\hline Process & $\begin{array}{c}t_{\text {TIs }} \\
(\text { COLLIER) }\end{array}$ & $\begin{array}{cc}t_{\mathrm{gen}} & t_{\mathrm{TCs}} \\
\text { (single helicity) }\end{array}$ & $\begin{array}{l}t_{\text {gen }} \quad t_{\mathrm{TCs}} \\
\text { (partial hel. sum) }\end{array}$ & $\begin{array}{c}t_{\mathrm{gen}} t_{\mathrm{TCs}} \\
\text { (helicity sum) }\end{array}$ \\
\hline $\begin{array}{c}u \bar{u} \rightarrow W^{+} W^{-} g \\
\quad(\mathrm{QCD})\end{array}$ & $2.8 \mathrm{~ms}$ & $\begin{array}{cc}0.3 \mathrm{~s} & 0.6 \mathrm{~ms} \\
\text { (hel: } & -+-+- \text { ) }\end{array}$ & 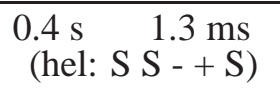 & $\begin{array}{c}1.6 \mathrm{~s} \\
\text { (hel: } \mathrm{S} \mathrm{S} \mathrm{S} \mathrm{S} \mathrm{S)}\end{array}$ \\
\hline $\begin{array}{c}u \bar{d} \rightarrow W^{+} g g g \\
(\text { QCD) }\end{array}$ & $130 \mathrm{~ms}$ & $\begin{array}{l}14 \mathrm{~s} \quad 14 \mathrm{~ms} \\
\text { (hel: }-+---- \text { ) }\end{array}$ & $\begin{array}{l}25 \mathrm{~s} \quad 76 \mathrm{~ms} \\
\text { (hel: S S - S S S) }\end{array}$ & $\begin{array}{l}52 \mathrm{~s} \quad 221 \mathrm{~ms} \\
\text { (hel: S S S S S S) }\end{array}$ \\
\hline $\begin{array}{c}u g \rightarrow u g Z \\
(\mathrm{EW})\end{array}$ & $8.2 \mathrm{~ms}$ & $\begin{array}{cc}0.5 \mathrm{~s} & 1.4 \mathrm{~ms} \\
\text { (hel: } & --- \text {-) }\end{array}$ & $\begin{array}{c}1.0 \mathrm{~s} \\
\text { (hel: S S S S -) }\end{array}$ & $\begin{array}{cc}2.2 \mathrm{~s} & 20.2 \mathrm{~ms} \\
\text { (hel: S S S S S) }\end{array}$ \\
\hline 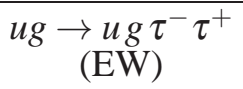 & $28 \mathrm{~ms}$ & $\begin{array}{cc}1.3 \mathrm{~s} & 2.5 \mathrm{~ms} \\
\text { (hel: }----+ \text { ) }\end{array}$ & $\begin{array}{l}2.0 \mathrm{~s} \quad 14.2 \mathrm{~ms} \\
\text { (hel: S S S S - +) }\end{array}$ & $\begin{array}{l}3.8 \mathrm{~s} \quad 29.0 \mathrm{~ms} \\
\text { (hel: S S S S S S) }\end{array}$ \\
\hline
\end{tabular}

Table 1: CPU time needed by RECOLA for the computation of sample processes with different helicity configurations (the "S" means that the sum over helicities has been taken for the corresponding particle). The computation has been performed with a processor Intel(R) Core(TM) i5-2400 CPU @ 3.10GHz.

current and each branch. The list of all needed TIs is also generated at this point. Currents differing just by the colour structures are identified and their colour coefficients are computed. In the second part all the TIs are computed (calling external libraries) as well as all currents for all polarizations, according to the skeleton derived in the generation part. Finally the TCs (i.e. the final currents) are contracted with the TIs to give the structure-dressed amplitudes $\mathscr{A}_{P}$, from which the squared amplitude summed over colour and polarization is calculated.

RECOLA allows the computation of tree and one-loop amplitudes in the full SM, including all counterterms [14] and rational parts of type R2 ${ }^{1}$ [15]. A consistent treatment of unstable particles is provided by using the complex-mass scheme [16]. On-shell renormalization is used for the EW sector, while the strong coupling constant is renormalized in the $\overline{\mathrm{MS}}$ scheme. The ultraviolet pole structure of one-loop amplitudes can be accessed numerically and for soft/collinear divergencies either dimensional or mass regularisation can be employed. For a given process at tree or one-loop level RECOLA allows to single out arbitrary powers in the strong coupling constant. In addition RECOLA calculates all colour- and spin-correlated amplitudes needed for the application of the dipole subtraction method [17]. The calculation of one-loop amplitudes relies on the external computation of the tensor integrals which is done with the COLLIER library [18].

In addition to an efficient treatment of colour, also the sum over helicity configurations has been optimized by avoiding recalculation of identical currents appearing in different helicity configurations together with the use of helicity conservation for massless fermions in the SM. The resulting code requires negligible amount of memory for executables, object files and libraries, while the RAM needed does not exceed 2 Gbyte even for complicated processes. For the sample processes in Table 1, the CPU time needed in the generation is of the order of some seconds, while the computation of the TCs takes at most few hundreds of milliseconds, usually of the same order of magnitude as the computation of the TIs with COLLIER.

\section{Conclusion}

We have presented RECOLA, an efficient code based on a one-loop generalization of Dyson-

\footnotetext{
${ }^{1}$ Since the loop currents are computed numerically, their indices are strictly 4-dimensional and the R2 part must be added separately.
} 
Schwinger recursion relations, which automatizes the computation of EW and QCD processes with elementary particles in the SM at NLO. The code has been used for the computation of EW corrections to the process $p p \rightarrow Z+2 j[9,19]$.

\section{Acknowledgments}

This work was supported in part by the Deutsche Forschungsgemeinschaft (DFG) under reference number DE 623/2-1.

\section{References}

[1] M. Ciccolini, A. Denner and S. Dittmaier, Phys. Rev. D 77 (2008) 013002 [arXiv:0710.4749 [hep-ph]].

[2] K. Arnold et al., Comput. Phys. Commun. 180 (2009) 1661 [arXiv:0811.4559 [hep-ph]].

[3] C. F. Berger et al., Phys. Rev. D 78 (2008) 036003 [arXiv:0803.4180 [hep-ph]].

[4] S. Badger, B. Biedermann and P. Uwer, Comput. Phys. Commun. 182 (2011) 1674 [arXiv:1011.2900 [hep-ph]].

[5] V. Hirschi et al., JHEP 1105 (2011) 044 [arXiv:1103.0621 [hep-ph]].

[6] G. Bevilacqua et al., arXiv:1110.1499 [hep-ph].

[7] G. Cullen et al., Eur. Phys. J. C 72 (2012) 1889 [arXiv:1111.2034 [hep-ph]].

[8] F. Cascioli, P. Maierhofer and S. Pozzorini, Phys. Rev. Lett. 108 (2012) 111601 [arXiv:1111.5206 [hep-ph]].

[9] S. Actis et al., JHEP 1304 (2013) 037 [arXiv:1211.6316 [hep-ph]].

[10] A. van Hameren, JHEP 0907 (2009) 088 [arXiv:0905.1005 [hep-ph]].

[11] F. J. Dyson, Phys. Rev. 75 (1949) 1736; J. S. Schwinger, Proc. Nat. Acad. Sci. 37 (1951) 452, Proc. Nat. Acad. Sci. 37 (1951) 455.

[12] A. Kanaki and C. G. Papadopoulos, Comput. Phys. Commun. 132 (2000) 306 [hep-ph/0002082]; F. Caravaglios and M. Moretti, Phys. Lett. B 358 (1995) 332 [hep-ph/9507237].

[13] A. Kanaki and C. G. Papadopoulos, hep-ph/0012004; F. Maltoni et al., Phys. Rev. D 67 (2003) 014026 [hep-ph/0209271].

[14] A. Denner, Fortsch. Phys. 41 (1993) 307 [arXiv:0709.1075 [hep-ph]].

[15] G. Ossola, C. G. Papadopoulos and R. Pittau, JHEP 0805 (2008) 004 [arXiv:0802.1876 [hep-ph]]; P. Draggiotis et al., JHEP 0904 (2009) 072 [arXiv:0903.0356 [hep-ph]]; M. V. Garzelli, I. Malamos and R. Pittau, JHEP 1001 (2010) 040 [Erratum-ibid. 1010 (2010) 097] [arXiv:0910.3130 [hep-ph]]; H. -S. Shao, Y. -J. Zhang and K. -T. Chao, JHEP 1109 (2011) 048 [arXiv:1106.5030 [hep-ph]].

[16] A. Denner et al., Nucl. Phys. B 724 (2005) 247 [Erratum-ibid. B 854 (2012) 504] [hep-ph/0505042].

[17] S. Catani and M. H. Seymour, Nucl. Phys. B 485, 291 (1997) [Erratum-ibid. B 510, 503 (1998)] [hep-ph/9605323].

[18] A. Denner and S. Dittmaier, Nucl. Phys. B 658 (2003) 175 [hep-ph/0212259], Nucl. Phys. B 734 (2006) 62 [hep-ph/0509141], Nucl. Phys. B 844 (2011) 199 [arXiv:1005.2076 [hep-ph]]; A. Denner, S. Dittmaier and L. Hofer, in preparation.

[19] A. Denner et al., these proceedings 